\title{
Dispersion of carbon nanotube (CNT) in bismaleimide(BMI) to prepare CNT/BMI foam
}

\author{
Xiao-Li Liu, ${ }^{1,2, a^{*}}$ \\ ${ }^{1}$ AVIC(Aviation Industry Corporation of China) Composite Corporation Ltd.,Beijing 100030, People's \\ Republic of China \\ ${ }^{2}$ AVIC (Aviation Industry Corporation of China)Composites Center, Beijing 10030, People's \\ Republic of China \\ ax|_nanliu@163.com
}

\begin{abstract}
Keywords: dispersion; carbon nanotube; bismaleimide foam
Abstract. Bismaleimide(BMI) structural foams employing carbon naotubes (CNTs) as functional fillers were prepared in this paper. Physical dispersion methods, such as mechanical mixing, ball milling and calendering process (three-roll milling), were adopted to disperse CNTs in the bismaleimide matrix to foam. The effects of the dispersion method on viscosity, bubble morphology were studied. The results indicated that, three-roll milling can more efficiently separate individual CNTs from the agglomerates compared with the other two methods. Because of more individual CNT to restraining the BMI chains, which exhibited the highest viscosity, the most irregular cell shape.
\end{abstract}

\section{Introduction}

Bismaleimide (BMI) foam [1,2] is one of the most important thermosetting foam, because of its thermal insulation properties, low weight, and low cost. Carbon nanotubes (CNTs) are lightweight materials, that have strong microwave absorption properties in the $\mathrm{GHz}$ frequency range[3,4]. Nanometer-scale diameter CNTs can avoid the limitation of large-sized conventional conductivity fillers, and their extremely large aspect ratio allows low loading of fillers to provide the desired electrical conductivity without sacrificing other inherent properties of the polymer. Therefore, CNTs are ideal fillers for preparing microwave-absorbing functional composites.

Numerous research efforts have been directed toward producing CNT/polymer composites for functional and structural applications[5,6,7]. However, even after a decade of research, the full potential of employing CNTs as reinforcements has been severely limited because of the difficulties associated with the dispersion of entangled CNT during processing and the poor interfacial interaction between CNTs and the polymer matrix. The nature of the dispersion problem for CNTs is rather different from those of other conventional fillers[8], such as spherical particles and carbon fibers, because CNTs are characteristic of small diameters in nanometer scale with high aspect ratios (>1000). Consequently, their surface area is extremely large. In addition, commercialized CNTs are supplied in the form of heavily entangled bundles, resulting in inherent difficulties in dispersion.

The approaches often used to disperse CNTs are optimum physical blending and functionalization-based dispersion. The surface modification and functionalization of CNTs can enhance their interaction with the polymer matrix, thus improving their dispersion in the matrix. Some of the methods for optimum physical blending are ultrasonication, shear mixing, calendering, ball milling, stirring, and extrusion. Chen et al.[9] synthesized epoxy/CNT nanocomposites in various ways to examine the effects of dispersion methods on their tribological properties. The dispersion and mixing methods used include dual asymmetric centrifuge, sonication, and hand mixing. Wear resistance improves with increasing effort placed into dispersion: sonication has a positive effect, and sonication plus dual asymmetric centrifugation has been proven to be even better. The results showed that the wear resistance increased with improved dispersion and integrity of the CNTs.

A foam employing CNT as reinforcement with BMI resin as matrix was prepared in this paper. The effects of physical dispersion methods (mechanical stirring, ball milling, and calender) on viscosity, bubble morphology and cell size were discussed. 


\section{Experimental}

\section{Materials}

The multi-wall CNTs were Flo Tube 9000 supplied by Tiannai Corporation (Beijing, China), with average length of $10 \mu \mathrm{m}$ and average diameter of about $11 \mathrm{~nm}$, and produced through catalytic vapor deposition process. Azodicarbonamide (ADC) purchased from the Letai Chemical Plant (Beijing, China) with decomposition temperature of $215^{\circ} \mathrm{C}$ and generated gas amount $210 \mathrm{~mL} / \mathrm{g}$ was used as the blowing agent. BMI was prepared in our laboratory.

\section{Sample Preparation}

Mechanical stirring method: CNTs and BMI resin were carefully weighed and mixed in a beaker with a propeller at a certain mixing speed (300rpm) for $20 \mathrm{~min}$.

Ball milling method: BMI and CNTs were dissolved in acetone and the blend was placed into a ball mill jar with stainless steel balls. The ball-milling process was carried out at $300 \mathrm{rpm}$ for a certain period (up to $48 \mathrm{~h}$ ). Afterward, the acetone was evaporated until a constant weight was achieved.

Three-roll milling method (calendering process): CNTs were manually stirred into the melt resin, and then the pre-dispersed suspension was placed onto the rolls. The suspensions were produced using a three-cycle program with the same distances $(0.5 \mu \mathrm{m})$ between the rolls. The speed for the apron roll was set to $300 \mathrm{rpm}$.

The obtained CNT/BMI nanocomposites through different dispersion methods were used for foaming. CNT/BMI foams were prepared through pre-polymerization and foaming. The suspension was heated to $140{ }^{\circ} \mathrm{C}$ and maintained at this temperature for $30 \mathrm{~min}$. Afterward, $5 \mathrm{wt} \%$ ADC was added to the melted prepolymer with constant stirring. The mixture was placed into a preheated mold $\left(180^{\circ} \mathrm{C}\right)$ for foaming and curing. Details about the foaming technique can be also found in earlier publications[1,2].

\section{Viscosity}

The changes in viscosity were determined at constant temperature of $140{ }^{\circ} \mathrm{C}$ using an AR 2000 (TA Instruments). The measurements were carried out at constant angular frequency of $1.0 \mathrm{rad} / \mathrm{s}$, with $1 \%$ strain.

\section{Scanning electron microscopy observations}

The fractured cross-sections of the BMI/CNT nanocomposites with different dispersion methods were gold sputtered and observed under a scanning electron microscope (SEM; Camscan 3100, Britain).

\section{Results and discussion}

\section{Dispersion of CNTs in the BMI matrix}

Fig. 1 shows the configuration of CNTs, in which the CNTs exhibited the characteristic of nanoscale-sized diameter with high aspect ratio $\left(10^{3}\right)$, and thus, extremely large surface area. CNTs are in the form of heavily entangled bundles mainly because of their tendency to agglomerate and entangle due to the strong van der Waal bonds, which become predominant as the CNT length increase. Subsequently, the lack of functional sites on the CNT surface also complicates the dispersion issue. To disperse the entangled CNTs and improve the interfacial interaction between CNTs and BMI matrix, three different physical dispersion routes to disperse CNTs homogeneously with the matrix were investigated. 

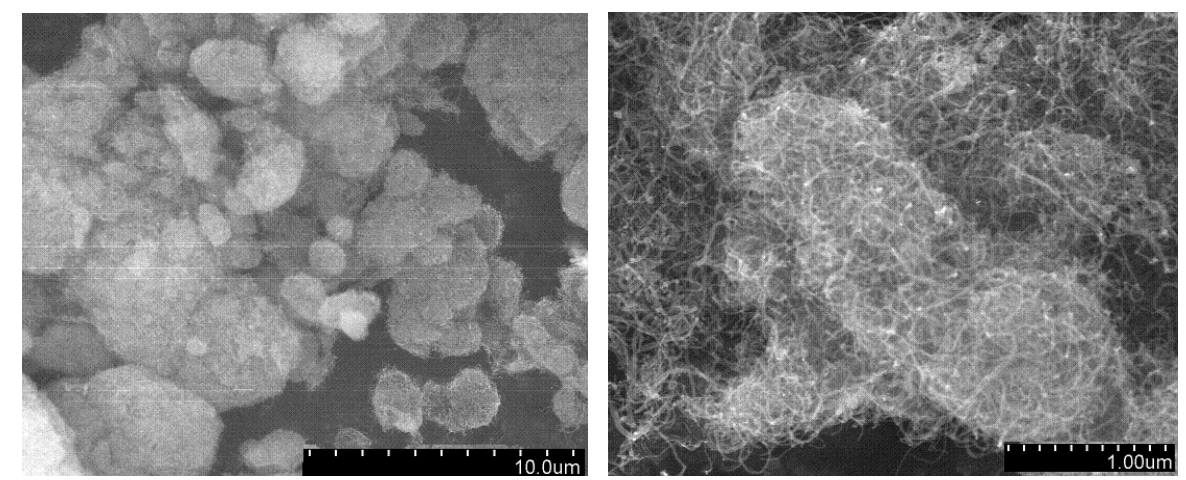

Fig 1 SEM images of the carbon nanotubes

Fig. 2 shows the SEM of the fractured cross-section of CNT/BMI nanocomposites prepared by different mixing methods. For the mechanical stirring method, large CNTs agglomerates (about $5 \mu \mathrm{m}$ to $10 \mu \mathrm{m}$ ) were observed in the composites, as shown in Fig 2(a). Although shear force and mixing energy are produced during melt mixing, CNT agglomerates can still remain entangled because of the strong intermolecular van der Waals interactions among the nanotubes. For the ball milling method, the CNT agglomerates are smaller (about $2 \mu \mathrm{m}$ to $3 \mu \mathrm{m}$ ), indicating the presence of relatively thin CNT bundles and partially disentangled CNTs inside the BMI matrix, as shown in Fig 2(b). During the milling, high pressure is generated locally because of the collision between the tiny, rigid balls in a concealed container, which partially disentangled the CNTs from the agglomerates. As shown in Fig 2(c), CNTs are uniformly dispersed in the BMI matrix using three-roll milling, and nearly no aggregates are observed, which indicated that the amount of CNTs is individually separated from the CNT agglomerates. The calender employs high shear force created by rollers to mix, disperse, and homogenize CNTs/BMI. However, the agglomerates can be destroyed by shearing force. Big CNT agglomerates mixed with BMI can be effectively opened by high shear force created by the calender.

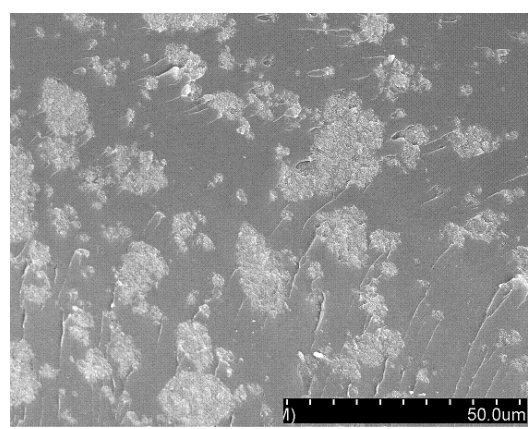

(a)

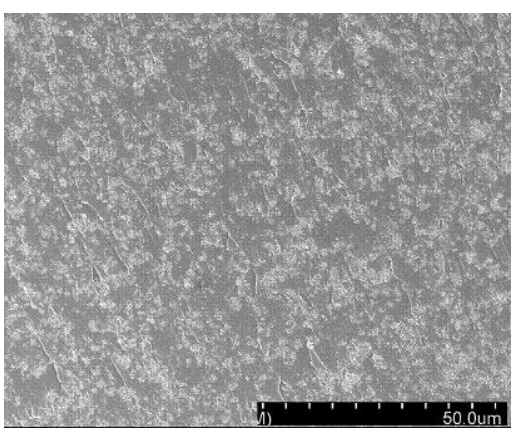

(b)

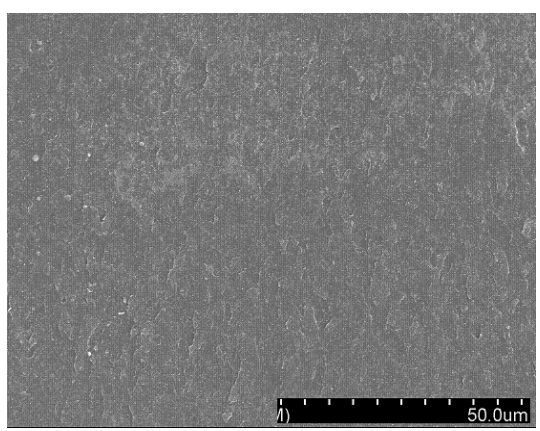

(c)

Fig 2 SEM image of the fracture surface of $1 \mathrm{wt} \%$ CNTs/BMI with different dispersion methods

(a) Mechanical stirring method; (b) Ball milling; (c) Three-roll milling

\section{Viscosity of foaming matrix with different dispersion methods}

The viscosity of BMI and CNT/BMI matrix at constant temperature $\left(140{ }^{\circ} \mathrm{C}\right)$ is shown in Fig. 3 . The CNTs increase the viscosity of the BMI matrix; however, the increasing effect is affected by the dispersion methods. At the same time interval, the sample prepared by three-roll milling exhibited the highest viscosity and the one prepared by mechanical stirring represented the lowest viscosity. The SEM results showed that the calender can effectively open CNT entanglements and individually separate more CNTs from the agglomerates. More individual CNTs can effectively restrain the relaxation of BMI chains in the nanocomposites, so the viscosity is much higher than the others. 


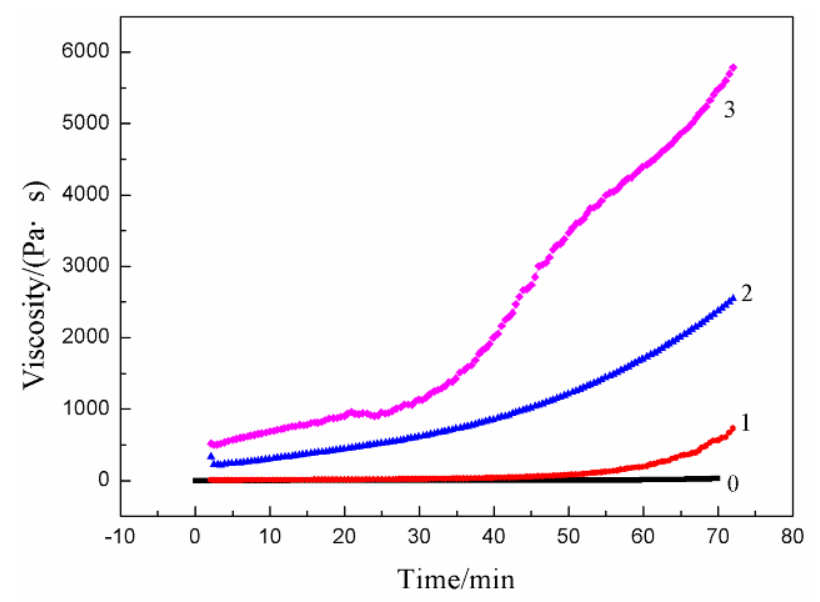

Fig 3 Viscosity of BMI and $1 \mathrm{wt} \% \mathrm{CNT} / \mathrm{BMI}$ prepared by different dispersion methods (0-BMI resin matrix; 1- CNT/BMI mechanical stirring; 2- CNT/BMI ball milling; 3- CNT/BMI three-roll milling)

\section{Morphology of BMI foams with different dispersion methods}

The effect of dispersion methods on the CNT/BMI foam morphology and the cell size distribution can be seen in Fig. 4. The cell size prepared by mechanical stirring method is about $0.38 \mathrm{~mm}$ and its distribution is more uniform than that of the others. The cell sizes are 0.56 and $0.69 \mathrm{~mm}$ for the foams prepared by ball milling and three-roll milling, and both their cell size distributions are not as uniform as the one prepared by mechanical stirring. This finding demonstrates that the morphology of the cell structure prepared by mechanical stirring is more uniform and nearly not affected by the presence of the CNTs. When the composites dispersed by mechanical stirring are foaming, blowing agent ADC generates gas, and the gas swells out isotropically because of the homogenous matrix, which is basically the same as the foaming mechanism of plastic foams and sponges[10]. However, for the ball milling and three-roll millings methods, the gas cell expansion may be anisotropic because of the incorporation of more individual CNTs. Individual CNTs has high aspect ratio and is anisotropic. More individual carbon nanotubes can effectively restrain the relaxation of the BMI chains in the nanocomposites, so the bubble morphology extensively changed. Meanwhile, the cell sizes decreased with the addition of nanoscale particles into the polymers. This finding is attributed to the fact that more bubbles start to nucleate concurrently, resulting in less gas for bubble growth, leading to a reduction of bubble size. The cell sizes increased with increasing individual CNTs in the BMI matrix, indicating that CNTs cannot serve well as heterogeneous nucleating agents during the foaming process.

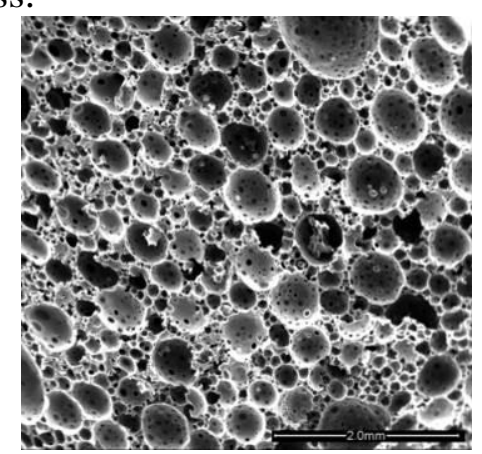

(a)

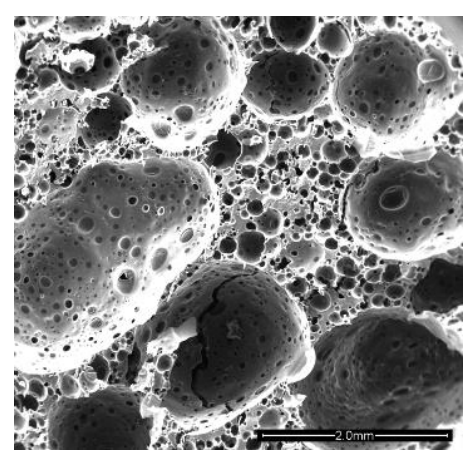

(b)

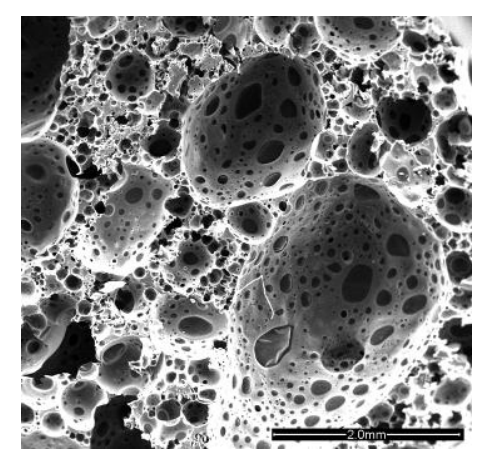

(c)

Fig4 SEM images of CNT/BMI foams and cell size distributions prepared by different dispersion methods

( $1 \mathrm{wt} \%$ CNTs; density, $160 \mathrm{~kg} / \mathrm{m}^{3}$, the cell sizes are $0,38 \mathrm{~mm}, 0.56 \mathrm{~mm}, 0,69 \mathrm{~mm}$, respectively) (a) Mechanical Stirring; (b) Ball Milling, cell size; (c) Three-Roll Milling 


\section{Conclusions}

Three-roll milling can more efficiently separate individual CNTs from the agglomerates compared with the other two methods.

More individual CNT to restraining the BMI chains for the one prepared by three-roll milling exhibited the highest viscosity, the most irregular cell shape.

\section{References}

[1] X.L. Liu, H.J. Lu, L.Y. Xing, Effect of prepolymerization time on morphology and properties of epoxy modified bismaleimide foams ,Int. Polym. Proc. 5,(2012) 617-625.

[2] X.L. Liu, H.J. Lu, L.Y. Xing, Study on micro-morphology and structure control of bismaleimide foams, J. Mater. Eng. 8,(2012)83-87.

[3] Ch.Ch.Zeng, N. Hossieny, C. Zhang, B. Wang, Synthesis and processing of PMMA carbon nanotube nanocomposite foams, Polymer, 51,(2010) 655-664.

[4] D.X.Yan, K. Dai, Zh.D. Xiang, Zh.M.Li, X Ji, W.Q. Zhang, Electrical conductivity and major mechanical and thermal properties of carbon nanotube filled polyurethane foams, J. Appl. Polym. Sci. 120,(2011), 3014-3019.

[5] P.Ch.Ma, N.A.Siddiqui, G.Marom, J.K.Kim, Dispersion and functionalization of carbon nanotubes for polymer based nanocomposites: A review,Compos.: Part A41 (2010), 1345 -1367 .

[6] G.R.Kasaliwal, S.Pegel, A.Goldel, P.Potschke, G. Heinrich, Analysis of agglomerate dispersion mechanisms of multiwalled carbon nanotubes during melt mixing in polycarbonate,Polymer 51(2012), 2708-2720.

[7] G.Gkikas, N.M.Barkoula, A.S. Paipetis, Effect of dispersion conditions on the thermo-mechanical and toughness properties of multi walled carbon nanotubes reinforced epoxy, Compos.: Part B, 43(2012), 2697-2705 .

[8] Zh. Zhou, Sh.F.Wang, Y.Zhang, Y.X. Zhang, Effect of Different carbon fillers on the properties of PP composites: comparison of carbon black with multiwalled carbon nanotubes,J. Appl. Polym. Sci. 102(2006),4823-4830.

[9] H.Y.Chen, O.Jacobs,W.Wu,G.Rudiger, B.Schadel. Effect of dispersion method on tribological properties of carbon nanotube reinforced epoxy resin composites, Polym. Test.26(2007),351-360.

[10] G.Lin, X.J.Zhang, L. Liu, J.Ch.Zhang, Q.M.Chen, L.Q. Zhang, Study on microstructure and mechanical properties relationship of short fibers/rubber foam composites,Eur. Polym. J. 40(2004) 1733-1742. 